\title{
A CHARACTERIZATION OF MAXIMAL CONNECTED SPACES AND MAXIMAL ARCWISE CONNECTED SPACES
}

\author{
BRADD CLARK AND VICTOR SCHNEIDER
}

(Communicated by Doug Curtis)

\begin{abstract}
The concept of a maximal connected space was developed by J. P. Thomas and maximal connected expansions of the reals were discovered by Guthrie, Stone, Wage, and Simon. This paper gives necessary and sufficient conditions for a space to be maximal connected. Also necessary and sufficient conditions for the join of two connected topologies to be connected are given in the special case that one of the topologies is atomic. Finally the concept of a maximal arcwise connected topology is given and used to show that every connected simplicial complex admits both a maximal arcwise connected extension and a maximal connected extension.
\end{abstract}

1. Introduction. In 1968 the concept of a maximal connected topology was introduced by J. P. Thomas in [7]. In [8] Thomas pointed out that any maximal connected space must be a M.I. space (A space is said to be M.I. if, and only if, every dense set is open.) Guthrie, Stone and Wage [4] and Petr Simon [6] gave a sufficient but not necessary condition to construct a maximal connected topology that contains the usual topology on the reals. The purpose of $\S 2$ is to characterize maximal connected spaces. We shall also consider the difficulty in obtaining maximal connected group topologies. In particular, we shall show that no connected M.I. group topology exists on $\mathbf{R}^{n}$.

A topology of the form $T(A)=\{\phi, A, X\}$ where $A$ is a proper subset of $X$ is called an atomic topology. This is because such a topology will be an atom in the lattice of all topologies on $X$. $\S 3$ will give necessary and sufficient conditions for a topology of the form $\tau \vee T(A)$ to be connected given that $\tau$ is connected. $\S 4$ will consider the concept of maximal arcwise connected spaces. A space $(X, \tau)$ is said to be maximal arcwise connected if, and only if, it is arcwise connected and every strictly finer topology fails to be arcwise connected. We shall show that every simplicial complex admits a finer maximal arcwise connected topology and a strictly finer maximal connected topology. This will generalize the work of Guthrie, Stone and Wage [4] and Simon [6].

\section{Nearly maximal and maximal connected spaces.}

DEFINITION. $(X, \tau)$ is nearly maximal connected if and only if $(X, \tau)$ is connected and for all regular open sets $V$ (i.e., $V=\operatorname{int}(\bar{V})$ ) and for all $x \in \partial V$ where $\partial V$ denotes the boundary of $V$ in $X$ relative to $\tau$, there exists a neighborhood $U_{1}$ of $x$ and an open nontrivial set $U_{2}$ such that $U_{1} \cap V \cap U_{2}=\varnothing$ and $U_{2} \cup\{x\}$ is closed.

The maximal singular expansions of $\mathbf{R}$ described in [4] provide examples of nearly maximal connected spaces that fail to be M.I. On the other hand, let $\tau^{\prime}$ be

Received by the editors February 25, 1987 and, in revised form, October 12, 1987.

1980 Mathematics Subject Classification (1985 Revision). Primary 54D05; Secondary 54A10. 
an ultrafilter of dense in the usual topology $\tau$, subsets of $\mathbf{R}$. Then $\tau \vee \tau^{\prime}$ will be an example of $\mathrm{M}$. I. space which fails to be nearly maximal connected.

THEOREM 1. $(X, \tau)$ is maximal connected if and only if it is nearly maximal connected and M.I.

ProOF. If $(X, \tau)$ is maximal connected, then as already pointed out, $(X, \tau)$ is M.I. Let $V$ be regular open and let $x \in \partial V$. Since $V \cup\{x\} \notin \tau$, then if $\tau^{\prime}$ is the topology generated by $\tau$ and $V \cup\{x\}, \tau^{\prime}$ is not connected. If $U$ is a nontrivial clopen set in $\tau^{\prime}$, then either $U$ or $X-U$ is an element of $\tau$. Call the nontrivial clopen set of $\tau^{\prime}$ that is in $\tau, U_{2}$. Since $U_{2}$ is not closed in $\tau$, but is closed in $\tau^{\prime}$, some of the $\tau$-limits points of $U_{2}$ must not be $\tau^{\prime}$-limit points of $U_{2}$. But $x$ is the only point in $X$ with a $\tau^{\prime}$-neighborhood base that is different from its $\tau$-neighborhood base. Therefore, $U_{2} \cup\{x\}$ is closed in $\tau$ and there exists a $\tau$-neighborhood $U_{1}$ of $x$ such that $U_{1} \cap(V \cup\{x\}) \cap U_{2}=\varnothing$. Thus $\tau$ is nearly maximal connected.

Now suppose that $\tau$ is M.I. and nearly maximal connected. Let $\tau \subset \tau^{\prime}$ and $U \in \tau^{\prime}-\tau$. If $\operatorname{Int}_{\tau}(U)=\varnothing$, then $U$ is closed in $(X, \tau)$ since $\tau$ is M.I. Hence $\tau^{\prime}$ fails to be connected. If $\operatorname{Int}_{\tau}(U) \neq \varnothing$ let $V_{1}=\operatorname{Int}_{\tau}(U)$. Since $U-\operatorname{cl}_{\tau}\left(V_{1}\right)$ is open in $\tau^{\prime}$ and has empty interior in $\tau$, it is either empty or $\left(X, \tau^{\prime}\right)$ is not connected. Hence we may assume that $V_{1} \subset U \subset \operatorname{cl}_{\tau}\left(V_{1}\right)$.

Let $V=\operatorname{Int}_{\tau} \operatorname{cl}_{\tau}\left(V_{1}\right)$. Then $\operatorname{cl}_{\tau} V=\operatorname{cl}_{\tau}\left(V_{1}\right)$ and so $V=\operatorname{Int}_{\tau} \operatorname{cl}_{\tau}(V)$. Since $\left(X, \tau^{\prime}\right)$ is M.I., if $U$ is an open set between $V$ and $\bar{V}=\operatorname{cl}_{\tau^{\prime}}(V)$, then any set between $V$ and $U$ is open. Since $U \notin \tau$ and $(X, \tau)$ is M.I., we have that $U \not \subset V$. So $V \cup U \in \tau^{\prime}-\tau$ and $V \cup U \subset \bar{V}$. Hence we may assume that $V \subset U$. Also for all $x \in U-V$ we have that $V \cup\{x\} \in \tau^{\prime}$ Since $V=\operatorname{Int}_{\tau} \operatorname{cl}_{\tau}(V)$, there exists a nontrivial open set $U_{2}$ and a neighborhood $U_{1}$ of $x$ such that $U_{1} \cap U_{2} \cap V=\varnothing$ and $U_{2} \cup\{x\}$ is closed in $\tau$, and hence closed in $\tau^{\prime}$. But $(V \cup\{x\}) \cap U_{1}$ is a $\tau^{\prime}$-neighborhood of $x$ which misses $U_{2}$. Thus $U_{2}$ is closed in $\tau^{\prime}$ and hence $\left(X, \tau^{\prime}\right)$ is not connected. Therefore, $(X, \tau)$ is maximal connected.

D. R. Anderson has developed a technique for extending a connected topology to a connected M.I. [1]. Using the language of lattice theory, Anderson's technique can be described as follows:

Let $\tau$ be a topology and $\mathscr{C}$ the collection of all dense subsets of $\tau$. Let $F$ be a filter of sets contained in $\mathscr{C}$ and $F \cup\{\varnothing\}=\tau_{F}$. Anderson proved that $\tau \vee \tau_{F}$ is connected if and only if $\tau$ is connected and M.I. if, and only if, $F$ is an ultrafilter. We shall prove that if $\tau$ is nearly maximal connected, then $\tau \vee \tau_{F}$ is maximal connected whenever $F$ is an ultrafilter.

THEOREM 2. Let $F$ be a filter of dense sets in $\tau$. If $\tau$ is nearly maximal connected, then $\tau \vee \tau_{F}$ is nearly maximal connected. Furthermore, $\tau \vee \tau_{F}$ fails to be a regular space whenever $\tau_{F} \nsubseteq \tau$.

Proof. Let $V \cap D$ with $V \in \tau$ and $D \in \tau_{F}$ be a typical basis element for $\tau \vee \tau_{F}$. Let $x$ be a $\tau$-limit point of $V$. Then if $x \in W \cap D^{\prime}$ with $W \in \tau$ and $D^{\prime} \in \tau_{F}$, we have that $D \cap D^{\prime}$ is dense in $\tau$ and $\varnothing \neq W \cap V \in \tau$. So $x \in \overline{V \cap D}$. Thus $\operatorname{cl}_{\tau}(V) \subset \overline{V \cap D}$. Since $\tau \vee \tau_{F}$ is finer than $\tau$, we also have $\bar{V} \subset \operatorname{cl}_{\tau}(V)$. Thus $\bar{V} \subset \operatorname{cl}_{\tau}(V) \subset \overline{V \cap D} \subset \bar{V}$ and therefore $\operatorname{cl}_{\tau}(V)=\overline{V \cap D}=\bar{V}$.

Now suppose that $U=\bigcup_{a \in \Gamma}\left(V_{a} \cap D_{a}\right)=\operatorname{int}(\bar{U})$. We have that $\bigcup_{a \in \Gamma} V_{a} \subset$ $\bigcup_{a \in \Gamma} \bar{V}_{a} \subset \bar{U}$. Hence $\bigcup_{a \in \Gamma} V_{a} \subset \operatorname{int}(\bar{U})=U$. But $U=\bigcup_{a \in \Gamma} V_{a} \cap D_{a} \subset \bigcup_{a \in \Gamma} V_{a}$. 
Therefore, $U=\bigcup_{a \in \Gamma} V_{a} \in \tau$. Thus $U$ satisfies the nearly maximal connected condition in $\tau$ and hence in the finer topology $\tau \vee \tau_{F}$.

Let $\tau^{\prime}$ be a maximal connected extension of $\tau$. Let $D \in \tau_{F}$ and $x \in D$ which fails to be in the $\tau$-interior of $D$. Let $x \in U \cap D^{\prime}$ be a typical basis element of $\tau^{\prime}$ with $U \in \tau$ and $D^{\prime}$ dense in $\tau$. Since $\overline{U \cap D^{\prime}}=\bar{U}$ we have that $\overline{U \cap D^{\prime}} \subsetneq D$.

If $(G, \tau)$ is a connected group topology, then $\tau$ must lie in a fairly narrow range of the collection of connected topologies in the lattice of all topologies on $G$. A moment's reflection will show that with the exception of the indiscrete topology, $\tau$ cannot be a hyperconnected topology. (A space is said to be hyper connected if, and only if, every nontrivial open set is dense.) However, if $G$ has $\mathbf{R}^{1}$ as a homomorphic image, then $G$ does not support a maximally connected group topology.

THEOREM 3. If $\tau$ is a connected group topology on $G$ and $G$ has $\mathbf{R}^{1}$ as a homomorphic image, then $\tau$ cannot be M.I.

ProOF. Suppose the theorem is false. If $S$ is a subgroup of $G$, then either $S=G$ or int $S=\varnothing$ since $\tau$ is connected. But if int $S=\varnothing$, then $G-S \in \tau$ and thus $S$ is closed.

Let $G=\mathbf{R}^{1}$ and $H$ be a linear complement to $Q$ when $\mathbf{R}^{1}$ is regarded as a vector space over $Q$. Since $H$ is not dense in $\tau$, we have that the quotient topology on $\mathbf{R}^{1} / H \approx Q$ associated with $\tau$ cannot be the indiscrete topology. But the indiscrete topology on $Q$ is the only connected group topology that exists on $Q$.

Now suppose that $h: G \rightarrow \mathbf{R}^{1}$ is an homomorphism. The associated quotient topology $\tau^{\prime}$ on $\mathbf{R}^{1}$ will have the property that dense subsets are open. But if $H$ is a subgroup of $\mathbf{R}^{1}$, it will be closed in $\tau^{\prime}$ since $p^{-1}(H)$ is a proper subgroup of $G$, whenever $H$ is a proper subgroup of $\mathbf{R}^{1}$. Hence the same arguments as above work.

3. Atomic extensions. Let $X$ be a set and $L(X)$ the collection of all topologies on $X$. Birkhoff pointed out in [2] that $L(X)$ can be made into a lattice when set inclusion is used as a partial ordering. Using lattice notation, one can ask, "When will $\tau_{1} \vee \tau_{2}$ be connected given that $\tau_{1}$ and $\tau_{2}$ are connected topologies?" This section will provide an answer to this question in a special case.

We shall assume throughout that $\tau$ is a connected topology on $X$. The notation $T(S)$ will stand or the atomic topology $\{\varnothing, S, X\}$ where $S$ is a proper subset of $X$. If $S \subset X$, we shall let $\bar{S}$ denote the closure of $S$ in $\tau$.

Let $A$ be a proper subset of $X$ and let $\left\{B_{\lambda} \mid \lambda \in \Gamma\right\}$ be the collection of components of $\bar{A}$. Let $A_{\lambda}=B_{\lambda} \cap A$. Of course, it is possible that $A_{a}=\varnothing$ for some $a \in \Gamma$. If $\Lambda$ is a subset (not necessarily proper) of $\Gamma$ let $A_{1}=\bigcup_{a \in \Lambda} A_{a}$ and $A_{2}=\bigcup_{\beta \in \Gamma-\Lambda} A_{\beta}$. We will call $\left\{A_{1}, A_{2}\right\}$ a decomposition of $A$. The decomposition $\left\{A_{1}, A_{2}\right\}$ is good if, and only if, $A_{1} \cap \bar{A}_{2}=\varnothing$.

THEOREM 4. $\tau \vee T(A)$ is disconnected if, and only if, there exists $U \in \tau$ and a good decomposition of $A$ into $\left\{A_{1}, A_{2}\right\}$ such that $U \cup A_{1}$ is a nontrivial closed set in $\tau \vee T\left(A_{2}\right)$.

ProOF. If $\tau \vee T(A)$ is disconnected, then there exists a separation of $X$ of the form $\left\{U \cup\left(U_{1} \cap A\right), V \cup\left(V_{1} \cap A\right)\right\}$ where $\left\{U, U_{1}, V, V_{1}\right\} \subset \tau$. If we let $U_{1} \cap A=A_{1}$, then clearly $A_{1}$ and $A_{2}=A-A_{1}$ will form a good decomposition. Also $U \cup A_{1}$ must be a nontrivial closed set in $\tau \vee T\left(A_{2}\right)$. 
This leads to two interesting corollaries.

COROLlaRY 2. If $\bar{A}$ is connected, then $\tau \vee T(A)$ is disconnected if, and only if there exists a $U \in \tau$ such $U \cup A$ is a nontrivial closed set in $\tau$.

COROLlaRY $3[\mathbf{1}]$. If $\bar{A}=X$, then $\tau \vee T(A)$ is connected.

Suppose that $\tau_{1}$ and $\tau_{2}$ are connected topologies, but that $\tau_{1} \vee \tau_{2}$ is a disconnected topology. One might conjecture that there exists $A \in \tau_{2}$ such that $\tau_{1} \vee T(A)$ is a disconnected topology. In general, however, this is not the case.

Let $\tau$ denote the usual topology on $\mathbf{R}$ and let $f: \mathbf{R} \rightarrow \mathbf{R}$ be a bijection. Let $\tau_{f}=\{f(U) \mid U \in \tau\}$. Then $\tau_{f}$ will be homeomorphic but not equal to $\tau$ whenever $f$ fails to be continuous in the usual topology. If $f$ is a noncontinuous automorphism of $\mathbf{R}$, then every nontrivial open set in $\tau_{f}$ is dense in $\tau$. Thus if $A \in \tau_{f}$, we have $\tau \vee T(A)$ is connected. But as Ellen Clay proved in [3], $\tau \vee \tau_{f}$ will be disconnected whenever $\tau_{f} \neq \tau$.

4. Maximal arcwise connected topologies. Following Kuratowski [5] we shall call a subspace homeomorphic to the unit interval with noncut points $x$ and $y$ an arc between $x$ and $y$.

DEFINITION. A topology $\tau$ is said to be a maximal arcwise connected topology if and only if it is arcwise connected and any topology $\tau^{\prime}$ with $\tau \subseteq \tau^{\prime}$ and $\tau \neq \tau^{\prime}$ fails to be arcwise connected.

THEOREMS 5. $(X, \tau)$ is a $T_{2}$ maximal arcwise connected space if, and only if, between every pair of points $\{x, y\}$, there exists a unique arc and $\tau$ is the coherent topology generated by this collection of unique arcs.

ProOF. Let $(X, \tau)$ be a maximal arcwise connected topology. Suppose that there are two arcs, $A_{1}$ and $A_{2}$, between $x$ and $y$. If $A_{1} \neq A_{2}$, then there is a point $z \in A_{1}-A_{2}$. Let $h: I \rightarrow A_{1}$ be a homeomorphism with $h(0)=x$ and $h\left(\frac{1}{2}\right)=z$. Let $\tau^{\prime}=\left\{\phi, X-h\left(\left[0, \frac{1}{2}\right)\right), X\right\}$. Since $h\left(\left[\frac{1}{2}, 1\right]\right) \cup A_{2}$ is an arcwise connected subspace of $\left(X, \tau \vee \tau^{\prime}\right)$ we have that $x, y$, and $z$ all belong to the same path component of $\left(X, \tau \vee \tau^{\prime}\right)$. Let $w \in X$ and let $A_{3}$ be an arc from $w$ to $z$ in $(X, \tau)$. Since $A=h\left(\left[0, \frac{1}{2}\right)\right) \cup h\left(\left[\frac{1}{2}, 1\right]\right) \cup A_{2}$ is arcwise connected in $\tau \vee \tau^{\prime}$ and $A_{3} \cap A \neq \varnothing$, we have that $w$ is in the same path component as $x, y$, and $z$. So $\left(X, \tau \vee \tau^{\prime}\right)$ is arcwise connected. Since this contradicts $\tau$ being a maximal arcwise connected topology, we must conclude that there is a unique arc between any two points in $(X, \tau)$. Since the topology on each arc is determined and the coherent topology on the collection of arcs is the finest topology that can be placed on $X$ consistent with these subspace topologies, we are done. The other direction is clear.

COROLlARY. If $(X, \tau)$ is a $T_{2}$ maximal arcwise connected space, then $(X, \tau)$ is neither M.I. nor nearly maximal connected.

THEOREM 6. If $(X, \tau)$ is a connected simplicial complex, then there exist topologies $\tau^{\prime}$ and $\tau^{\prime \prime}$ with $\tau \subseteq \tau^{\prime} \subseteq \tau^{\prime \prime}$ such that $\tau^{\prime}$ is a maximal arcwise connected topology and $\tau^{\prime \prime}$ is a maximal connected topology.

ProOF. Let $T$ be a maximal tree contained in the one-skeleton $K_{1}$ of $X$. If $A$ is a 1-simplex in $K_{1}-T$ and $x$ is a vertex of $A$, let $\tau(A)=\{\varnothing,(X-A) \cup\{x\}, X\}$ and $\tau_{1}=\left(\bigvee_{A \in K_{1}-T} \tau(A)\right) \vee \tau$. $\tau_{1}$ induces a maximal arcwise connected topology 
on $K_{1}$. For each $n$-simplex $S$ of $X$ with $n>1$, choose a vertex $v \in S \cap T$. For each point $x \in \operatorname{int} S$, there exists a unique ray $R(x)$ emanating from $v$ through $x$. $\tau^{\prime}$ is the coherent topology generated from the natural topology placed on each ray $R(x)$ and the topology $\tau_{1}$ on $K_{1}$.

Since a maximal connected topology containing the usual topology can be placed on any interval of the reals, it is possible to place such a topology on $K_{1}$ containing $\tau_{1}$ and such a topology on each ray $R(x)$ containing the usual topology. The topology $\tau^{\prime \prime}$ will be generated by placing the associated coherent topology on $X$.

\section{REFERENCES}

1. D. R. Anderson, On connected irresolvable Hausdorff spaces, Proc. Amer. Math. Soc. 16 (1965), 463-466.

2. G. Birkoff, On the combination of topologies, Fund. Math. 26 (1936), 156-166.

3. E. Clay, Joins of Euclidean orbital topologies, Master's Thesis, University of Southwestern Louisiana, 1986.

4. J. A. Guthrie, H. E. Stone and M. L. Wage, Maximal connected expansions of the reals, Proc Amer. Math. Soc. 69 (1978), 159-163.

5. C. Kuratowski, Topology, vol. 1, Academic Press, New York, 1966.

6. P. Simon, An example of maximal connected Hausdorff space, Fund. Math. 100 (1978), 157-163.

7. J. Thomas, Maximal connected topologies, J. Austral. Math. Soc. 8 (1968), 700-705.

8. __ Maximal connected Hausdorff spaces, Pacific J. Math. (1975), 581-583.

Department of Mathematics, University of Southwestern Louisiana, LaFAYETTE, LOUISIANA 70504 